\title{
Beyond size exclusion: Online liquid chromatography for BioSAXS
}

\author{
M. Brennich ${ }^{a}$, S. Hutin ${ }^{b}$, K. Weinhäupl ${ }^{b}$, P. Schanda ${ }^{b}$, P. Pernot ${ }^{c}$ \\ ${ }^{a}$ EMBL, Grenoble Outstation, 71 Avenue des Martyrs, Grenoble, France \\ ${ }^{b}$ Institut de Biologie Structurale, 71 Avenue des Martyrs, Grenoble, France \\ ${ }^{c}$ ESRF-The European Synchrotron, 71 Avenue des Martyrs, Grenoble, France
}

Small angle X-ray scattering of proteins and nucleic acids (BioSAXS) can provide a wealth of information on their structural configuration in solution [1]. For many, if not most, bio-macromolecules, however, this configuration is not unique, but an ensemble of different structures and stoichiometries. Therefore, despite recent advances in instrumentation and analysis tools, obtaining useful results on individual states can still be challenging.

Online purification of samples directly prior to the measurement can reduce the structural variability. For this, online size exclusion chromatography (SEC) has been established as a standard approach that not only helps to obtain SAXS curves for differently sized species within a sample, but can also indicate conformational changes within a single species [2].

However, changes in structure and shape are not always accompanied by sufficiently large changes in overall size for efficient separation by SEC. Additionally, SEC requires high initial concentrations, in which the oligomeric state of interest can be unfavourable. Affinity based gradient elution techniques separate based on biochemical properties such as surface charge and effectively decouple final and initial concentrations. In this presentation we show two applications of the technique: Using online ion-exchange chromatography we acquired good quality SAXS data of a viral helicase complex while maintaining its monomeric state at moderate salt concentrations [3]. Online nickel affinity chromatography separated the complex of a chaperone protein with its target from the individual components, enabling insights into the complex arrangement.

These techniques are available for interested users as part of the standard setup at ESRF BM29.

[1] M. Graewert and D. Svergun (2013), Curr. Op. Struct. Biol. 23, 748-754.

[2] G. David \& J. Pérez (2009), J. Appl. Cryst. 42, 892-900.

[3] S. Hutin, et al. (2016), Acta Cryst. D72, 1090-1099. 